\title{
Vertex Reconstruction and Deep Learning Applications in JUNO
}

\section{Ziyuan Li*, Zhengyun You, Yumei Zhang, Jiang Zhu, Shu Zhang}

School of Physics, Sun Yat-Sen University, Guangzhou, P.R.China

E-mail: liziyuan3email.sysu.edu.cn

\section{(On behalf of the JUNO collaboration)}

The Jiangmen Underground Neutrino Observatory (JUNO), currently under construction in the south of China, will be the largest liquid scintillator (LS) detector in the world. JUNO is a multipurpose neutrino experiment designed to determine neutrino mass hierarchy, precisely measure oscillation parameters, and study solar neutrinos, supernova neutrinos, geo-neutrinos and atmospheric neutrinos. The central detector (CD) of JUNO contains 20,000 tons of LS and about 18,000 20-inch as well as 25,000 3-inch photomultiplier tubes (PMT). The energy resolution is expected to be $3 \% / \sqrt{E(\mathrm{MeV})}$. To meet the requirements of the experiment, two algorithms for the vertex reconstruction have been developed. One is the time likelihood method which utilizes the time and charge information of PMTs with good understanding of the complicated optical processes in the LS. The other is the deep learning method with the convolutional neural networks architecture, which is fast and avoids the need to consider in detail the optical processes. In general, similar performances of both methods are achieved. The deep learning method tends to give more accurate prediction near the detector border region, where the optical processes are more complicated.

XXIX International Symposium on Lepton Photon Interactions at High Energies - LeptonPhoton2019 August 5-10, 2019

Toronto, Canada

${ }^{*}$ Speaker. 


\section{JUNO Experiment}

JUNO[1] is a 20 kiloton LS detector. The inner diameter of the acrylic ball is $35.4 \mathrm{~m}$ (Fig. 1). When neutrinos go through the LS, some of them interact with the LS through inverse beta decay: the absorbed energy is released through scintillation photons. Depending on the interaction vertex, scintillation photons may go through a long distance before they are detected by the PMTs. During their propagation in the LS, these photons are more likely to be absorbed, reemitted, scattered, or reflected. Additionally, the refractive index is 1.49 for the LS and 1.33 for the pure water. The large difference of refractive indices between two materials results in the large effects of refraction and total reflection at the boundary of two materials, which will affect the time of flight in the LS for scintillation photons. Hence, the optical model is critical for the vertex resolution and is key to the success of the JUNO experiment.

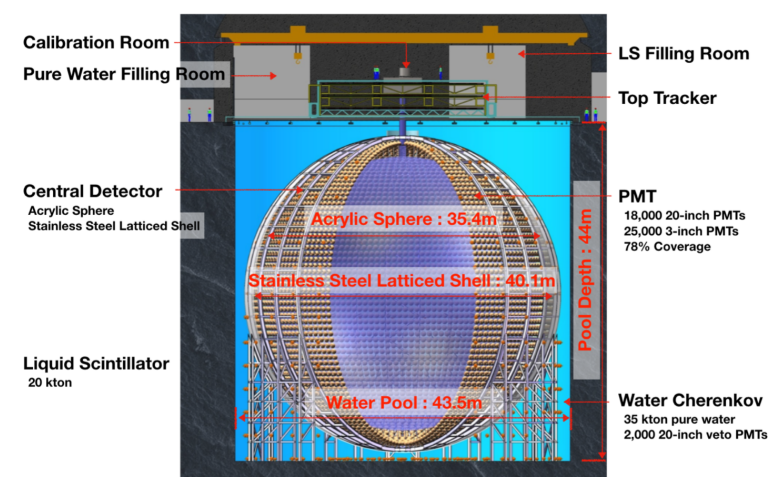

Figure 1: Schematic view of JUNO detector.

\section{Time Likelihood Vertex Reconstruction}

For point-like events, i.e. $e^{+}, e^{-}$or $\gamma$ which deposit energy in LS and emits photons with vertex position $\vec{r}_{0}$ and generation time $t_{0}$, the residual hit time variable is defined as:

$$
t_{i, r e s}\left(\vec{r}_{0}, t_{0}\right)=t_{i}-\text { tof }_{i}-t_{0}
$$

where $t_{i}$ is first hit time of $i^{t h}$ PMT and to $f_{i}$ is the time of flight for the scintillation photon.

The probability density function $f\left(t_{\text {res }}\right)$ reflects the dispersion time of scintillation light emission, together with additional effects introduced through scattering, absorption and re-emission, reflection and refraction of the photon on its way to the PMT, as well as the finite time resolution of the PMTs (Fig. 2 (Left)). The probability density function for a single photoelectron is derived from a Monte Carlo (MC) simulation, while the probability density function of the first photon to reach a PMT out of $n$ photoelectrons could be calculated through equation 2.2 :

$$
f_{n}\left(t_{\text {res }}\right)=n f\left(t_{\text {res }}\right)\left(\int_{t_{\text {res }}}^{\infty} f(x) d x\right)^{n-1}
$$

A likelihood function is defined and can be minimized to get the best vertex position:

$$
\mathscr{L}\left(\vec{r}_{0}, t_{0}\right)=-\sum_{i} \ln \left(f_{n}\left(t_{\text {res }}\right)\right)
$$




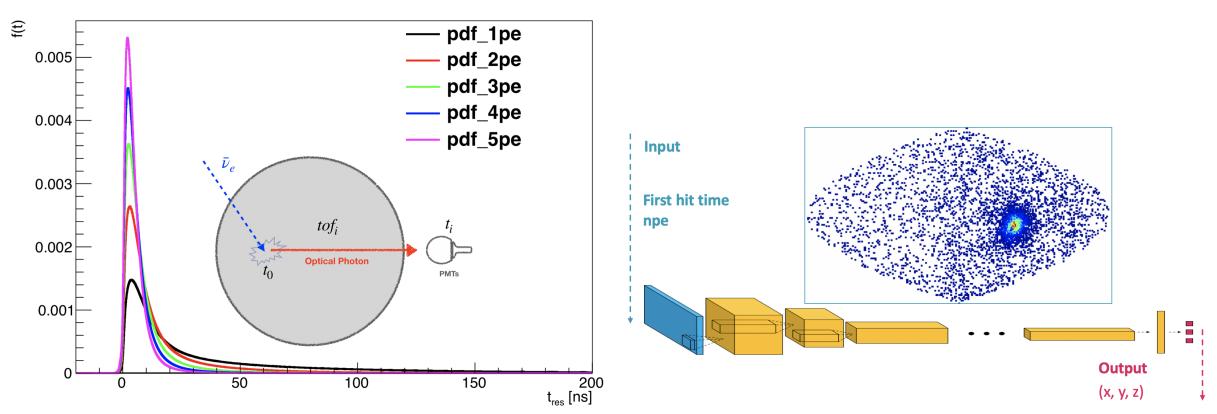

Figure 2: $f_{n}\left(t_{\text {res }}\right)$ distribution (Left). Projection of detector and schematic view of deep learning (Right).

An event display software has been designed for the JUNO[2, 3, 4]. It is an essential tool to display the detector and event data for better understanding of the physics processes in the detector. The trajectory of the deposited energy and produced scintillation photons can be clearly seen, which is very helpful for vertex reconstruction study.

\section{Deep Learning Vertex Reconstruction}

One of the most popular algorithms for deep learning is supervised learning with convolutional neural networks (CNN)[5]. CNN is specially developed for image analysis, by reducing the parameter space with convolution. A CNN is composed of an input layer, an output layer, and many hidden layers in between. With such structures, CNN is able to learn features from given images during the training procedure and then used to classify newly input images by recognizing their features with the same algorithm.

Since CNN needs images as the input, we project PMTs on CD surface onto a two-dimensional plane and build a $230 \times 126 \times 2$ matrix, as shown in Fig. 2 (Right). We generate 200 million MC $e^{+}$ with energy from 1 to $10 \mathrm{MeV}$ and uniformly distributed within $\mathrm{CD}$ as a training dataset. For each event, we use the hit time of the first arrival photon and number of photoelectrons on each PMTs as input variables, and the energy deposited position $(x, y, z)$ from MC simulation as output variables. Different layer structures have an impact on model performance. In our study, after optimization, we use ResNet model with 50 convolutional layers and approximately 35 million parameters.

\section{Performance}
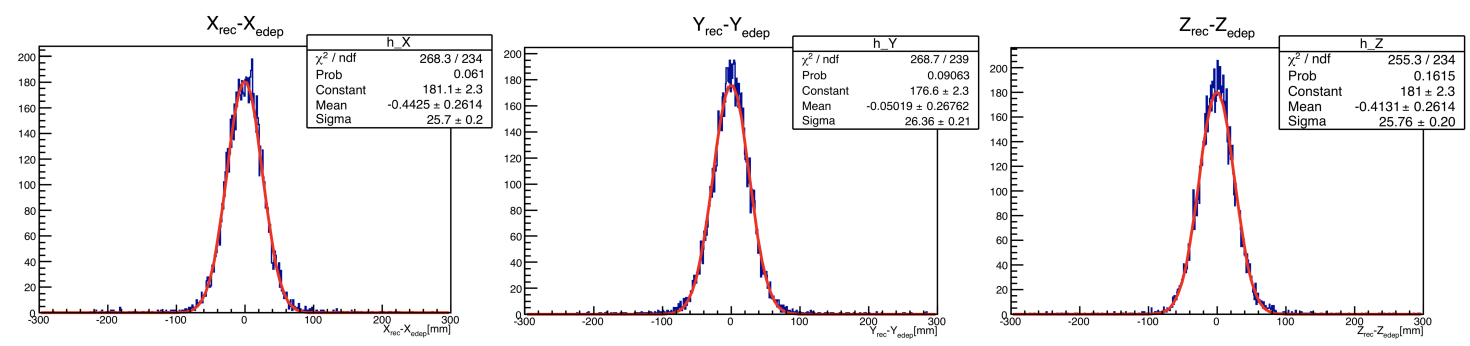

Figure 3: An example of reconstructed vertex resolution in each direction for $e^{+}$with $E_{\text {edep }}=4.5 \mathrm{MeV}$. 
By comparing the reconstructed event position and the deposited energy position in the MC simulation, the reconstructed vertex resolution could be estimated by a gaussian fit (see Fig. 3). The mean values are consistent with zero and the resolutions are similar in the three directions.

The vertex resolution as a function of energy for two methods is shown in Fig. 4 (Left). In general, similar performances of both methods are achieved: $\sim 60 \mathrm{~mm}$ at $1 \mathrm{MeV}$ and $\sim 25 \mathrm{~mm}$ above $8 \mathrm{MeV}$. The deep learning method consumes much less CPU time and tends to give more accurate predictions especially near the detector border region, where the optical processes are more complicated, as shown in Fig. 4 (Right).
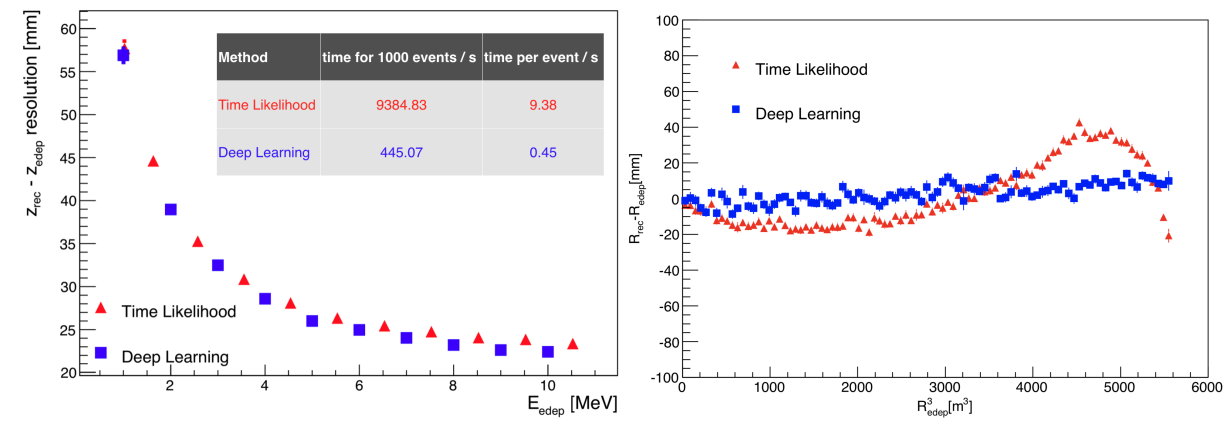

Figure 4: Vertex Resolution vs. Energy (Left). Vertex Bias Mean vs. $R^{3}$ for $e^{+}$with $E_{\text {edep }}=4.5 \mathrm{MeV}$ (Right).

\section{Conclusions}

Two algorithms to reconstruct vertex position have been developed based on MC simulation. They achieve similar performances in the energy region relevant to reactor neutrinos. Further studies are required to incorporate the detector responses, such as the PMTs transit time spread and dark noise, for a better description of the real experimental data.

\section{Acknowledgments}

This work is supported by the National Natural Science Foundation of China (11675275, 11805294, 11975021), the China Postdoctoral Science Foundation (2018M631013) and the Fundamental Research Funds for the Central Universities (19lgpy268).

\section{References}

[1] F. An et al. [JUNO Collaboration], Neutrino physics with JUNO, J.Phys. G 43 (2016) no.3, 030401.

[2] K. Li, Z. You, Y. Zhang et al., GDML based geometry management system for offline software in JUNO, Nucl. Instrum. Meth. A 908 (2018) 43-48.

[3] Z. You, K. Li, Y. Zhang et al., A ROOT based event display software for JUNO, JINST 13 (2018) T02002.

[4] J. Zhu, Z. You, Y. Zhang et al., A method of detector and event visualization with Unity in JUNO, JINST 14 (2019) no.01, T01007.

[5] R. Li, Z. You and Y. Zhang, Deep Learning for Signal and Background Discrimination in Liquid based Neutrino Experiment, J. Phys.: Conf. Ser. 1085 (2018) 042037. 